\title{
FLEXIBLE PARYLENE-VALVED SKIN FOR ADAPTIVE FLOW CONTROL
}

\author{
Nick Pornsin-Sirirak, Matthieu Liger, Yu-Chong Tai \\ Caltech Micromachining Laboratory \\ Electrical Engineering Department, California Institute of Technology \\ Pasadena, CA 91125 \\ Steve Ho and Chih-Ming Ho \\ Mechanical and Aerospace Engineering \\ University of California \\ Los Angeles, CA 90095
}

\begin{abstract}
This paper describes the first work of using wafer-sized flexible parylene-valved actuator skins (total thickness $\sim 20$ $\mu \mathrm{m})$ for micro adaptive flow control. The check-valved actuator skins feature vent-through holes with tethered valve caps on the membrane to regulate pressure distribution across the skins. The skins were integrated onto MEMS wings and were tested in the low-speed wind tunnel for aerodynamic evaluation. The test results have shown very significant effects on the aerodynamic performances. Compare to the reference MEMS wings (no actuators), both the lift and thrust of the parylene check-valved wings were improved by more than $50 \%$. This is the first experimental result to demonstrate that the application of MEMS actuator skins for flow control is very promising.
\end{abstract}

\section{INTRODUCTION}

From our previous study of MEMS wings for MAV applications [1], titanium-alloy wings with parylene membrane were fabricated to test unsteady aerodynamics mode of flapping flight. We have learned that the control of the wings' pressure distribution is very important in order to achieve an optimal aerodynamic performance. Thus, it is logical to fabricate devices on the membrane, passive or active, that can regulate the wing loading. To achieve this, we reported last year a chip-sized flexible parylene technology [2]. The more challenging next step, hence this work, is to make wafer-sized flexible parylene actuator membranes or "skins," and integrate these skins onto the MEMS wings. The goal is to distribute actuators on the wings to regulate pressure distribution during downstroke and upstroke. This concept is illustrated in Figure 1. The pneumatic actuation relies upon the wing loading during flapping. For each flapping cycle, the pressure loading either pushes the valve caps to open or close, controlling the air movement through the vent holes. The opening of the valves equalizes the pressure between the upper and the lower surfaces of the wings. When the valves are closed, the existence of the pressure difference can affect the aerodynamic performance, i.e., lift and thrust.

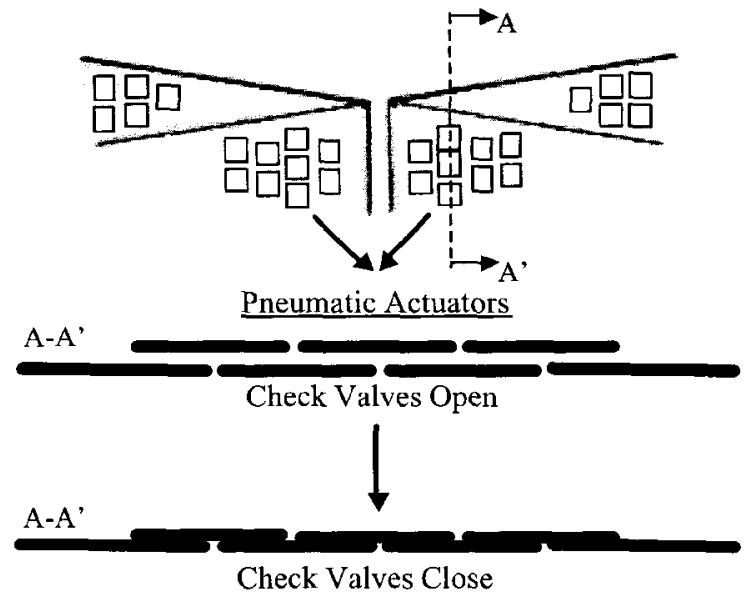

Figure 1: Integrated actuator MEMS wing concept

In a fluidic system, a check-valve is a device used to control liquid or gas flow in one direction. The valve is opened by a forward fluid flow and is closed by the reverse flow. It is analogous to a diode used to rectify the direction of current flow in an electric circuit. Recently, various structures of MEMS micro check-valve such as cantilevers, floats, gates, ring mesas, and membranes that were fabricated from silicon, metal, silicone, polyimide, and parylene have been developed [3-8]. However, they were mainly based on bulk micromachining of silicon or glass structure. There has been no previous work of check-valve presented using freestanding parylene skin before.

This paper describes the first flexible, wafer-sized parylene check-valved skin intended for micro adaptive flow control of the MEMS wings. Photoresist is used as a sacrificial layer to release the skin. We have also integrated anti-stiction technologies using amorphous silicon as an anti-stiction layer [9]. At the end of the process, dry gas etching of $\mathrm{BrF}_{3}[10]$ is used to etch the amorphous silicon to completely free the valve caps. Aerodynamic test results in a low-speed wind tunnel will be presented and analyzed. In addition, challenges and future work will be discussed. 


\section{DESIGN AND FABRICATION}

The parylene-valved skin consists of many small checkvalved actuators that are made of two layers of parylene membranes. Each layer of parylene is approximately $10-\mu \mathrm{m}$ thick. There are 107 dice on one wafer skin. On each individual die, there are 5 rows and columns of parylene check-valves. Each valve consists of a circular vent hole and a valve cap. Each vent hole is $1.5 \mathrm{~mm}$ apart. The vent holes and valve caps have diameters of $500 \mu \mathrm{m}$ and $900 \mu \mathrm{m}$, respectively. The valve cap is connected to the skin by four flexible reverse " $S$ " arms which allow the valve cap to open or close depending on the direction of the air flow. Once the check-valve is closed, the valve cap can cover the entire vent holes completely to minimize any air leakage. The fabrication process of the flexible parylene-valved skin is shown in Figure 2.

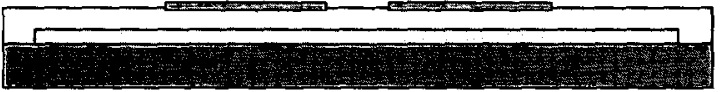

(a) Sacrificial PR; par1 $(10 \mu \mathrm{m})$; sputtered Si, pattern\#l(Si)

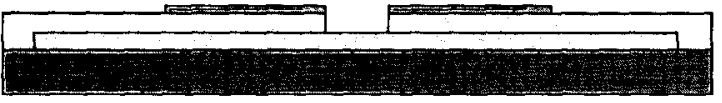

(b) Evap Al, pattern $\# 2(\mathrm{Al})$; etch parylene; strip $\mathrm{Al}$

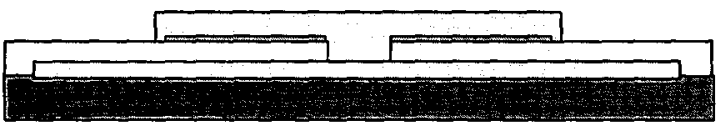

(c) Spin $2^{\text {nd }}$ sacrificial PR; pattern\#3(PR)

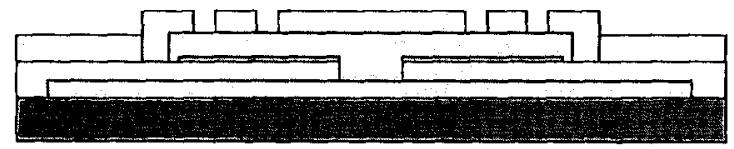

(d) Par2(10 $\mathrm{m})$; evap Al; pattern\#4(Al); etch parylene; strip Al

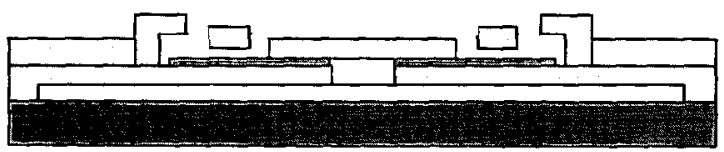

(e) Release PR; SAM

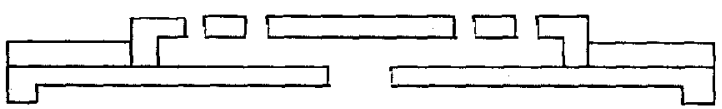

(f) BrF3; release the skin

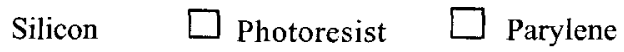

Figure 2: Summary of the fabrication process

The process begins with the first sacrificial layer of photoresist on a wafer. The resist is hardbaked at $120^{\circ} \mathrm{C}$ for 30 minutes to completely bake out the solvent. Next, the first layer of parylene is deposited and then roughened in the oxygen plasma. The wafer is then annealed at $120^{\circ} \mathrm{C}$ for 30 minutes to reduce stress in the parylene film. Afterwards, a thin film of amorphous silicon is deposited and patterned. This layer is used as an anti-stiction layer. Aluminum film is evaporated as a mask to pattern parylene. After parylene patterning, the sacrificial layer of photoresist is spun on and patterned. Then the top layer of parylene is deposited and patterned to form the valve caps. Next, the photoresist is released in an acetone bath to free the skin and then SAM layer is coated [11]. Finally, the amorphous silicon is etched in $\mathrm{BrF}_{3}$ gas to complete the fabrication process. Figure 3 shows the released parylene-valved actuator skin.

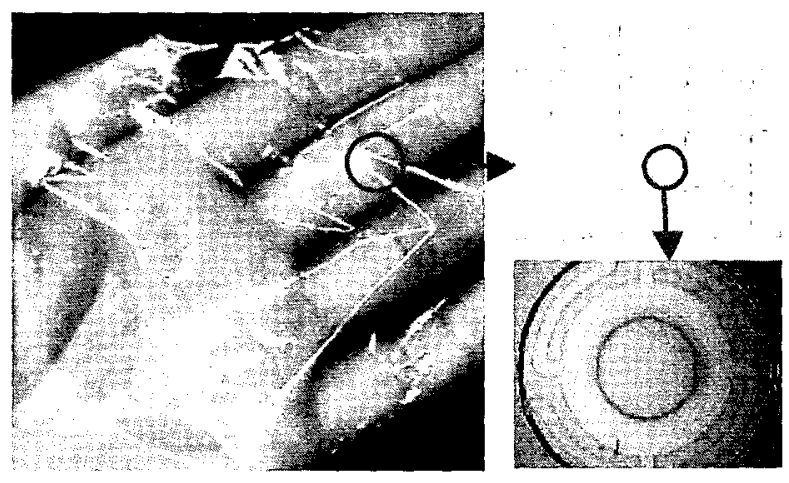

Figure 3: Flexible parylene-valved actuator skin

There are several processing challenges during the fabrication that are worth noted. For example, because the corner step of the vent hole is the thickness of the bottom parylene $(\sim 10 \mu \mathrm{m})$, it is important that the $2^{\text {nd }}$ sacrificial photoresist must be thick enough to cover this step corner. Otherwise the top and bottom parylene films will fuse together at the corner. This can prevent the valves from opening. Figure 4 displays other problems that are also encountered. Figure 4 (a) shows bubbles generated the $1^{\text {st }}$ sacrificial photoresist during the annealing of parylene film. This problem occurs because the $1^{\text {st }}$ sacrificial photoresist is not hardbaked long enough to get rid of the solvent. Thus the remedy is to hardbake the photoresist at $120{ }^{\circ} \mathrm{C}$ for at least 30 minutes. Figure 4 (b) shows the buckling of the thin amorphous silicon film due to the stress mismatch between the silicon and parylene films. By annealing the parylene film before the amorphous silicon deposition, the stress in parylene becomes tensile. The buckling cffect can be reduced. Lowering the deposition power also helps alleviate this problem. Finally, Figure 4 (c) shows the stiction of the valve after photoresist is released in acetone. After the skin is dry, the valves are stuck and cannot be opened. By having an amorphous silicon layer underneath the caps, dry etching of $\mathrm{BrF}_{3}$ gas can etch the thin silicon layer away and helps release the valves without any stiction. 


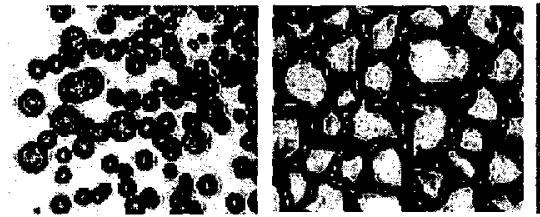

(a) Resist bubbles (b) Buckling

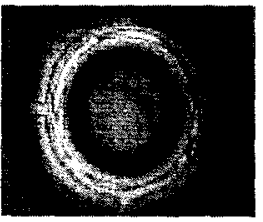

(c) Stiction
Figure 4: Examples of processing challenges

\section{RESULTS AND DISCUSSION}

The actuator skins are integrated onto the MEMS wings and tested in a low-speed wind tunnel as seen in Figure 5. In addition to the actuator skins, parylene pin-holed skins with $500-\mu \mathrm{m}$ hole diameter are also fabricated. The design of these skins is identical to those of the check-valved type except that there are no valve-caps. The pin-hole-integrated wings, as well as unmodificd MEMS wings, are used as references for comparison of the effect of the check-valved actuators. Table 1 summarizes the phase-average results for reference, pin-holed, and check-valved integrated MEMS wings. Figure 6 and 7 show the plots of lift and thrust forces of those wings. Phase averaging is the method used to achieve instantaneous lift and thrust measurements by collecting the entire lift and thrust history of a complete downstroke and upstroke cycle. It also reduces the noise levels as many flapping cycles are averaged. The final data is the result of the integration of the lift and thrust values over the entire period of flapping cycle.

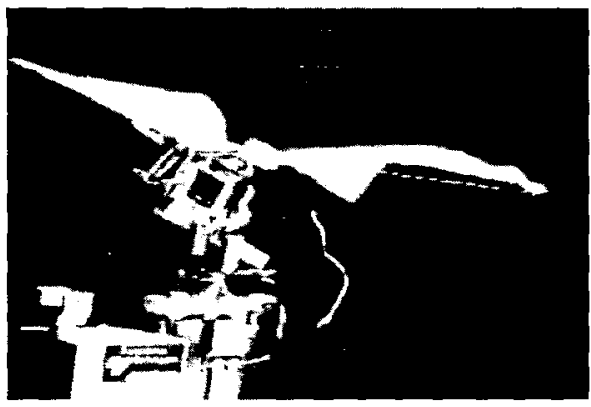

Figure 5: Wind tunnel aerodynamic test

Table 1: Summary of phase-average results

\begin{tabular}{||l|c|c|}
\hline Wing types & Lift $[\mathrm{gl}$ & Thrust [gl \\
\hline \hline Reference & 2.190 & 0.454 \\
\hline Pin-holes $(500-\mu \mathrm{m})$ & $1.376(-37 \%)$ & $0.223(-51 \%)$ \\
\hline $\begin{array}{l}\text { Check-valves open on } \\
\text { downstroke }\end{array}$ & $2.996(+37 \%)$ & $0.718(+58 \%)$ \\
\hline $\begin{array}{l}\text { Check-valves close on } \\
\text { downstroke }\end{array}$ & $3.358(+53 \%)$ & $0.663(+46 \%)$ \\
\hline
\end{tabular}
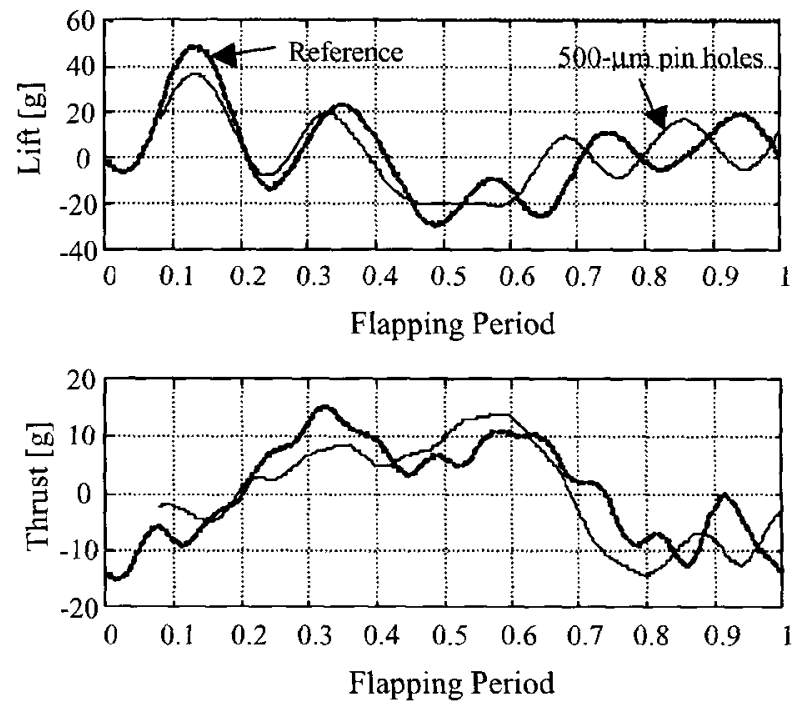

Figure 6: Phase-average results of the reference and pinholed integrated wings
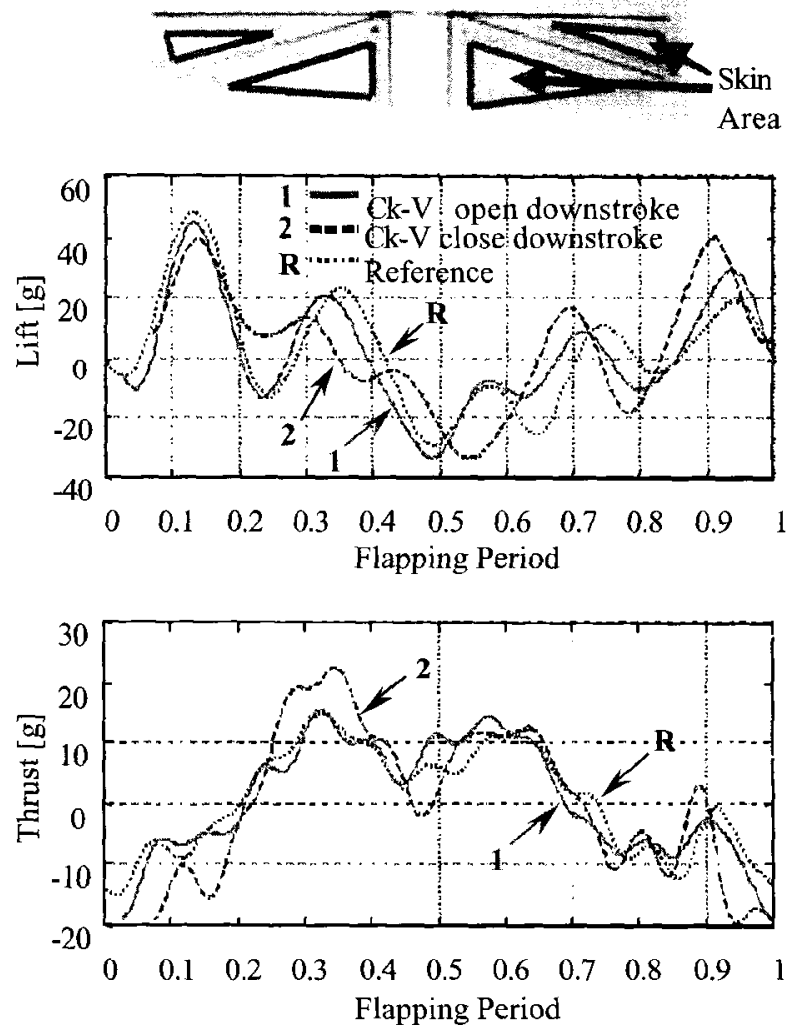

Figure 7: Aerodynamics and phase-average results of the check-valved integrated wings

The phase-average results of the reference and pinholed integrated wings in Figure 6 indicate that the pin- 
holed integrated wings do not perform as well as the reference wings. With only vent holes on the wings, the air can flow through the holes freely in both downstroke $(0-0.5)$ and upstroke (0.5-1) cycles. The holes equalize the pressure on the upper and lower surfaces of the wings. The wing loading is reduced in both upstroke and downstroke, hence the reduction in aerodynamic performance. The phaseaverage of lift and thrust are $37 \%$ and $51 \%$, respectively, lower than those of the unmodified reference wings.

On the other hand, the test results of the pneumatic check-valved type have shown a dramatic effect on the aerodynamic performance. The MEMS wings integrated with flexible parylene-valved actuator skin are shown in Figure 7. The actuators are placed in the leading-edge and trailing-edge areas where they are believed to significantly affect the aerodynamic performance of the wings. The check-valved actuators are mounted onto the MEMS wings in two different directions. First, the valves open during downstroke and close on upstroke. Second, the valves close during downstroke and open on the upstroke. Aerodynamic test results show lift has increased significantly by $37 \%$ and $53 \%$ for the first and second ways, respectively. Similarly, the thrust has also increased by $58 \%$ and $46 \%$, respectively. These numbers show tremendous improvements. The plots also show that there is a phase delay between opening and closing of the valves. The effect moderates the extreme lift peaks and in particular the negative lift peak that happens during the downstroke. The aerodynamic performance is highly dependent on the formation of the shedding vortices. Disturbing the leading-edge vortices will vary the pressure distribution and alter the aerodynamic performance. This delay also causes the upstroke peaks to change and phase shift relative to the unmodified reference wing.

\section{CONCLUSIONS}

We have successfully demonstrated the dramatic effect on the aerodynamic performance of the MEMS wings by integrating with parylene-valved skin. Compare to the reference MEMS wings, both lift and thrust of the parylenevalved wings, mounted such that the check-valves close during downstroke, can be improved by almost $50 \%$. This is the first experimental result to demonstrate the promising application of MEMS actuator skins for flow control.

This work also includes the novel anti-stiction technology that is crucial to release such large-area parylene actuator skin with the combined use of SAM, amorphous silicon layer, and $\mathrm{BrF}_{3}$ dry etching. The future work will include the design, fabrication, and test results of active check-valved electrostatic actuator skin.

\section{ACKNOWLEDGEMENTS}

The authors would like to thank DARPA (DABT63-98C-0005) and NSF ERC for their generous supports.

\section{REFERENCES}

[1] T. N. Pornsin-Sirirak, S.W. Lee, H. Nassef, J. Grasmeyer, Y.-C. Tai, C. -M. Ho, M. Keennon, "MEMS Wing Technology for a Battery-Powered Ornithopter," Proceedings of the $13^{\text {th }}$ IEEE Annual International Conference on MEMS 2000, Miyazaki, Japan, Jan. 23-27, 2000, pp. 799-804.

[2] T. N. Pornsin-Sirirak, T.-C. Tai, H. Nassef, C.-M. Ho, "Flexible Parylene Actuator for Micro Adaptive Flow Control," Proceedings of the $14^{\text {th }}$ IEEE Annual International Conference on MEMS 2001, Interlaken, Switzeland, Jan. 21-25, 2001, pp. 511-514.

[3] H. T. G. van Lintel, F. C. M. van de Pol, and A. Bouwstra, "Piezoelectric micropump based on micromachining of silicon," Sensors and Actuators, vol. 15 , no. 2, 1988, pp. 153-168.

[4] J. Tirén, L. Tenerz, and B. Hok, "A batch-fabricated non-reverse valve with cantilever beam manufactured by micromachining of silicon," Sensors and Actuators, vol. 18, 1989, pp. 389-396.

[5] M. Esashi, S. Shoji, and A. Nakano, "Normally closed microvalve and micropump fabricated on a silicon wafer," Proc. IEEE Micro Electro Mechanical Systems (MEMS '89), Salt Lake City, UT, Feb. 20-22, 1989, pp. 29-34.

[6] L. Smith and B. Hok, "A silicon self-aligned nonreverse valve," Digest of Tech. Papers, Int. Conf. on Solid-State Sensors and Actuators (Transducers 91), San Francisco, CA, June 24-27, 1991, pp. 1049-1051.

[7] S. Shoji, B. H. van der Schoot, N. F. de Rooij, and M. Esashi, "A study of a high-pressure micropump for integrated chemical analyzing systems," Sensors and Actuators, A32, 1992, pp. 335-339.

[8] X. Q. Wang, Q. Lin, and Y. -C. Tai, “A Parylene Micro Check Valve," The $12^{\text {th }}$ IEEE International Conference on Micro Electro Mechanical Systems (MEMS '99), Florida, U.S.A, Jan. 17-21, 1999, pp. 177-182.

[9] T. J. Yao, X. Yang, and Y. -C. Tai, "BrF 3 Dry Release Technology for Large Freestanding Parylene MEMS," Digest of Technical Papers, The $11^{\text {th }}$ International Conference on Solid-State Sensors and Actuators (Transducers '01), Munich, Germany, June 10-14, 2001, pp. 652-655.

[10] X. Q. Wang, X. Yang, K. Walsh, and Y. C. Tai, "GasPhase Silicon Etching with Bromine Trofluoride," Digest of Technical Papers, The 9th International Conference on Solid-State Sensors and Actuators (Transducers '97), Vol. 2, Chicago, IL, June 16-19, 1997, pp. 1505-1508.

[11] R. Maboudian, "Self-Assembled Monolayers as AntiStiction Coatings for Surface Microstructures," Digest of Technical Papers, The $10^{\text {th }}$ International Conference on Solid-State Sensors and Actuators (Transducer'99), Vol I, Sendai, Japan, June 7-10, 1999, pp. 22-25. 